\title{
Gangliocytic Paraganglioma: a Diagnostic Pitfall of Rare Neuroendocrine Tumor
}

\author{
Yoichiro Okubo ${ }^{1}$
}

Published online: 4 April 2017

(C) Springer Science+Business Media New York 2017

\section{Dear Editor:}

We wish to comment on the gangliocytic paraganglioma research. Since hypoxia-inducible factor $2 \alpha$ gain-of-function mutations were detected in gangliocytic paraganglioma (GP) [1], as well as paraganglioma and somatostatinoma [2], this neuroendocrine tumor may be relative tumors of paraganglioma and/or somatostatinoma. In fact, there are similar morphological and immunohistochemical features between paraganglioma and GP, except for the existence of ganglion-like cell in GP [3]. In addition, GP often shows positive immunoreactivity for somatostatin [3, 4]. To differentiate GP from paraganglioma, pathologists should consider the primary tumor site and the existence of ganglion-like cell (paraganglioma lacks ganglion-like cells, and it is very rare in the duodenum) [4]. On the other hand, morphological differences between GP and somatostatinoma make differentiation easy, and immunoreactivity for progesterone receptor and pancreatic polypeptide which is discussed below may be useful to differentiate them [5].

Meanwhile, the most important differential diagnosis of GP is neuroendocrine tumor (NET) G1. Indeed, the patients with GP may have a better prognosis than the patients with NET G1, but GP has often been misdiagnosed as NET G1 owing to its morphological similar findings and low proliferative activity $[4,5]$. Thus, we would like to emphasize the usefulness of immunohistochemical examinations using progesterone receptor and pancreatic polypeptide to differentiate them. Namely, typical epithelioid cell (a major component of GP) shows positive immunoreactivity with them, whereas tumor cell of NET G1 was negative for both immunohistochemistries $[4,5]$. We believe that these findings may be useful to differentiate GP from NET G1, including small specimen obtained from biopsy.

Acknowledgments This work was supported by a Grant-in-Aid for Scientific Research (\#17K08713) from the Ministry of Education, Culture, Sports, Science, and Technology of Japan and a research grant from the Kanagawa Cancer Center (\#28-07).

\section{References}

1. Zhuang Z, Yang C, Ryska A et al. HIF2A gain-of-function mutations detected in duodenal gangliocytic paraganglioma. Endocr Relat Cancer 23: L13-L16, 2016.

2. Zhuang Z, Yang C, Lorenzo F et al. Somatic HIF2A gain-of-function mutations in paraganglioma with polycythemia. N Engl J Med 367: 922-930, 2012.

3. Okubo Y, Wakayama M, Nemoto T et al. Literature survey on epidemiology and pathology of gangliocytic paraganglioma. BMC Cancer 11: 187, 2011.

4. Okubo Y, Yokose T, Motohashi O et al. Duodenal rare neuroendocrine tumor: clinicopathological characteristics of patients with gangliocytic paraganglioma. Gastroenterol Res Pract 2016: 5257312, 2016.

5. Okubo Y, Nemoto T, Wakayama M et al. Gangliocytic paraganglioma: a multi-institutional retrospective study in Japan. BMC Cancer 15: 269, 2015.
Yoichiro Okubo

yoichiro0207@hotmail.com

1 Department of Pathology, Kanagawa Cancer Center, 2-3-2, Nakao, Asahi-Ku, Yokohama, Kanagawa 241-8515, Japan 